\title{
Modification terms to the Black-Scholes model in a realistic hedging strategy with discrete temporal steps
}

\author{
Choi-Hong Lai *
}

\begin{abstract}
Option pricing models generally require the assumption that stock prices are described by continuous-time stochastic processes. Although the time-continuous trading is easy to conceive theoretically, it is practically impossible to execute in real markets. One reason is because real markets are not perfectly liquid and purchase or sell any amount of an asset would change the asset price drastically. A realistic hedging strategy needs to consider trading that happens at discrete instants of time. This paper focuses on the impact and effect due to temporal discretisation on the pricing partial differential equation (PDE) for European options. Two different aspects of temporal discretisation are considered and used to derive the modification or correction source terms to the continuous pricing PDE. First the finite difference discretisation of the standard Black-Scholes PDE and its modification due to discrete trading. Second the discrete trading leads to a discrete time re-balancing strategy that only cancels risks on average by using a discrete analogy of the stochastic process of the underlying asset. In both cases high order terms in the Taylor series expansion are used and the respective correction source terms are derived.
\end{abstract}

\section{Introduction}

An option pricing model requires the assumption that the underlying asset prices are described by continuous-time stochastic processes. The dynamics governing

\footnotetext{
${ }^{*}$ Department of Mathematical Sciences, University of Greenwich, UK. Corresponding author email: C.H.Lai@gre.ac.uk
} 
an underlying asset may be described by a stochastic differential equation (SDE) involving a volatility parameter, a drift coefficient and a stochastic process $d B$. This is the randomness involved in the SDE and is known as a Weiner process. In trading options or bonds, the main aim to ask for is how to achieve a fair price and how to protect financial risks when one wishes to buy or sell. Answer to this has been presented in [2] which essentially says that this risks from an option can be offset by a portfolio of assets that needs to continuously rebalanced. If no arbitrage is possible, the value of the option is the cost of this replication strategy. Theoretically the timecontinuous trading is easy to achieve. However it is difficult to execute it in real markets which are usually not perfectly liquid. Therefore the Black-Scholes PDE produces option pricing results using continuous time are inevitably to be different from a discretised Black-Scholes pricing results. The objective of this paper is to address this discrepency through the use of Taylor expansion leading to the concept of a correction source term in the Black-Scholes PDE for option pricing. The concept of consistency employed in finite difference methods in the analysis of discretised PDEs is employed in the first instance to demonstrate the effect of discrete trading due to temporal and spatial discretisation of the Black-Scholes PDE. A discrete application of the stochastic process govering the underlying asset is used in the second instance to analysis a discrete trading strategy through the use of average values.

The paper is organised as follow. Section 2 provides a brief overview of the continuous hedging and the development in various discrete hedging strategy. Section 3 discusses the modification to the Black-Scholes equation when a finite difference discretisation is used. Section 4 examines the Taylor expansion to include higher order expansion when the trading is not continuous. In both cases the correction source terms are derived leading to the modified pricing equation as described by the modified Black-Scholes PDE. Finally conclusions are drawn.

\section{Continuous Hedging}

The stochastic differential equation (SDE)

$$
d S=\mu S d t+\sigma S d B
$$

is the mathematical representation of a fundamental recipe for generating asset prices. Here $S$ denote the asset price, $\mu$ is the growth rate of the asset, $\sigma$ is the 
volatility, and $d B$ is a Weiner process. It should be noted that the above equation does not provide a deterministic path of the asset price $S$, but only its value in a probabilistic sense. Although real life asset prices are always recorded at discrete intervals of time, mathematical models for the valuation of option prices would become simpler and conceivable theoretically in continuous time limit $d t \rightarrow 0$ using Itô's lemma. The technicalities leading to Itô's lemma [9] involving the random term $d B$ rely on the limits, $d B^{2} \rightarrow d t, d B d t \rightarrow 0$ and $d t^{2} \rightarrow 0$, as $d t \rightarrow 0$, where the right arrows denote tends to. Using eqn (1) one can obtain $d S^{2} \rightarrow \sigma^{2} S^{2} d t$ as $d t \rightarrow 0$, and thus $d S^{k} \rightarrow 0$, for $k>2$.

Let $V(S, t)$ be a function of the asset price which is often known as the value of an option. Taylor expansion of $V(S+d S, t+d t)$ may be written as

$$
\begin{aligned}
V(S+ & d S, t+d t)=V(S, t)+\left(\frac{\partial V}{\partial S} d S+\frac{\partial V}{\partial t} d t\right) \\
& +\frac{1}{2}\left(\frac{\partial^{2} V}{\partial S^{2}} d S^{2}+\frac{\partial^{2} V}{\partial t^{2}} d t^{2}+2 \frac{\partial^{2} V}{\partial S \partial t} d S d t\right) .
\end{aligned}
$$

Thus the incremental change of the option value as $d t \rightarrow 0$ is given by

$$
d V=\left(\frac{\partial V}{\partial S} d S+\frac{\partial V}{\partial t} d t\right)+\frac{1}{2}\left(\frac{\partial^{2} V}{\partial S^{2}} d S^{2}+\frac{\partial^{2} V}{\partial t^{2}} d t^{2}+2 \frac{\partial^{2} V}{\partial S \partial t} d S d t\right)
$$

which is rearranged in the form of Itô's lemma as

$$
d V=\sigma S \frac{\partial V}{\partial S} d B+\left(\frac{\partial V}{\partial t}+\frac{1}{2} \sigma^{2} S^{2} \frac{\partial^{2} V}{\partial S^{2}}+\mu S \frac{\partial V}{\partial S}\right) d t
$$

In order to obtain the price of an option or other financial derivatives following the above SDE, standard assumptions [15] of a perfect market has to be used. These include no transaction costs, no dividends, no arbitrage, risk-free interest rate and known asset volatility. The risk-free interest rate is usually denoted as $r$ and it simply means that the return in a time $d t$ of a portfolio $\Pi$ is $r \Pi d t$. The porfolio constructed in the Black-Scholes analysis relies on one option with a number $\Delta$ of the underlying asset $S$.

$$
\Pi=V-\Delta S
$$

Since

$$
d \Pi \equiv r \Pi d t=d V-\Delta S,
$$

it is possible to offset the risk and eliminate the uncertainty in the option prices by setting

$$
\Delta=\frac{\partial V}{\partial S}
$$


This leads to the Black-Scholes partial differential equation (PDE)

$$
\frac{\partial V}{\partial t}+\frac{1}{2} \sigma^{2} S^{2} \frac{\partial^{2} V}{\partial S^{2}}+r S \frac{\partial V}{\partial S}-r V=0
$$

subject to suitable terminal and boundary conditions provides deterministic price of many options and derivatives.

Finite difference method is usually used in obtaining numerical solutions of (6) typically when the volatility is not a constant when closed form solutions do not exist [15][11][12]. Non-constant volatility is often discussed in the literature, including implied volatility [8] and volatility smile [5], just to name a couple of these. Computational efficiency based on parallel temporal domain methods [3][6][7] were also examined in the literature in order to provide faster solution processes to facilitate trading.

Although the pricing of options based on eqn (6) is simpler and conceivable theoretically, it certainly has two weaknesses. First the option value $V(S, t)$ derived from the equation depends on continuous trading which is effectively impossible. A modification to the Black-Scholes PDE would be able to take care of this weakness. Second discretisation schemes applied to eqn (6) would introduce discretisation errors into the numerical solution of the option pricing. Therefore suitable modification would be able to mitigate this weakness.

\section{Discrete Hedging}

It should be noted that real life asset prices are always recorded at discrete intervals of time. In the continuous hedging case the Black Scholes model in eqn (6) actually replicates the option payoff exactly by continuously rebalancing in time a portfolio consisting of the underlying asset and a risk-free option. A linear relationship between asset returns and the risks exists. However continuous trading becomes impossible when such relationship is nonlinear.

Early investigation into the effect of discrete hedging on option pricing using Black-Scholes model was considered by Derman ([4]). The study compared the replication of a single option using the Black-Scholes model for the lifetime of the option with the case that only a discrete number of rebalancing trades at regular intervals were permitted. On the other hand a discrete-time model was derived leading to a partial differential equation with similar structure as the Black-Scholes model and was used to partially hedge a single option. Wilmott also examined a 
minimum variance approach in order to partially hedge a single option with discretetime model. This resulted to a partial differential equation of the same structure $[14]$ as the Black-Scholes equation.

This paper examines two discrete aspects of the Black-Scholes equation as given in eqn (6). Section 4 examines the effect of finite difference discretisation of eqn (6) and the modification requires to mitigate the pricing problem due to discrete trading imposed as a result of the discretisation. The concept of consistency for the finite difference schemes is used to derive the modification. Section 5 examines the effect of trading due to discrete temporal steps involved in a discrete dynamics resembling the stochastic process as descibed by the SDE in eqn (1). In other words the modification to eqn (6) is built when trading cannot be done continuously. To simplify the analysis $\sigma$ and $\mu$ in eqn (1) is taken as constants in the sequel.

\section{Finite Difference Discretisation and its Effect}

Rewrite eqn (6) as

$$
\frac{\partial V}{\partial t}=-\left(\frac{1}{2} \sigma^{2} S^{2} \frac{\partial^{2} V}{\partial S^{2}}+r S \frac{\partial V}{\partial S}-r V\right) \equiv L_{B S} V
$$

defined in the domain $\Omega=\left[0, S_{\max }\right] \times[0, T]$ subject to suitable boundary and terminal conditions, where $S_{\max }$ is usually chosen as an integral multiple of the strike price. Finite difference methods are usually applied to eqn (7) in order to obtain a numerical solution. For the ease of exposition of the finite difference methfod, the domain has a uniformly distributed rectangular mesh with mesh point $\left(S_{i}, t_{n}\right)$, where $S_{i}=i \delta S, i=0,1,2, \ldots, M$, such that $\delta S=S_{\max } / M$, and $t_{n}=n \delta t$, $n=0,1,2, \ldots, N$, such that $\delta t=T / N$. In the current case the numerical scheme with a backward difference along the temporal axis and a central difference schme along the spatial axis is used. This results to the finite difference approximation to eqn $(7)$ at a typical nodal point $\left(S_{i}, t_{n}\right)$ as below.

$$
\frac{V_{i}^{n+1}-V_{i}^{n}}{\delta t}=-\left\{\frac{1}{2} \sigma^{2} S_{i}^{2} \frac{V_{i-1}^{n}-2 V_{i}^{n}+V_{i+1}^{n}}{\delta S^{2}}+r S_{i} \frac{V_{i+1}^{n}-V_{i-1}^{n}}{2 \delta S}-r V_{i}^{n}\right\},
$$

where $\delta t$ is the temporal discretisation step in the backward temporal finite difference scheme and $\delta S$ is the spatial discretisation step in the central finite difference scheme. The temporal discretisation step is in essence equivalent to the discrete trading time step as imposed by the above finite difference scheme. 
Taylor series expansion of $V_{i}^{n+1}$ leads to the series below with high order terms.

$$
\begin{gathered}
\frac{V_{i}^{n+1}-V_{i}^{n}}{\delta t}=\frac{\partial V\left(S_{i}, t_{n}\right)}{\partial t}+ \\
\frac{\delta t}{2} \frac{\partial^{2} V\left(S_{i}, t_{n}\right)}{\partial t^{2}}+\frac{\delta t^{2}}{6} \frac{\partial^{3} V\left(S_{i}, t_{n}\right)}{\partial t^{3}}+\frac{\delta t^{3}}{24} \frac{\partial^{4} V\left(S_{i}, t_{n}\right)}{\partial t^{4}}+\ldots,
\end{gathered}
$$

Differentiating eqn (7) with respect to $t$ leads to

$$
\frac{\partial^{2} V(S, t)}{\partial t^{2}}=L_{B S}\left(L_{B S} V\right)
$$

and again leads to

$$
\frac{\partial^{3} V(S, t)}{\partial t^{3}}=L_{B S}\left(L_{B S}\left(L_{B S} V\right)\right)
$$

Taylor series expansion of $V_{i+1}^{n}$ and $V_{i-1}^{n}$ lead to the series below including some high order terms.

$$
\begin{gathered}
\frac{V_{i+1}^{n}-V_{i-1}^{n}}{2 \delta S}=\frac{\partial V\left(S_{i}, t_{n}\right)}{\partial S}+ \\
\frac{\delta S^{2}}{3 !} \frac{\partial^{3} V\left(S_{i}, t_{n}\right)}{\partial S^{3}}+\frac{\delta S^{3}}{4 !} \frac{\partial^{4} V\left(S_{i}, t_{n}\right)}{\partial S^{4}}+\ldots,
\end{gathered}
$$

and

$$
\begin{gathered}
\frac{V_{i-1}^{n}-2 V_{i}^{n}+V_{i+1}^{n}}{\delta S^{2}}=\frac{\partial^{2} V\left(S_{i}, t^{n}\right)}{\partial S^{2}}+ \\
\frac{2 \delta S^{2}}{4 !} \frac{\partial^{4} V\left(S_{i}, t^{n}\right)}{\partial S^{4}}+\frac{2 \delta S^{4}}{6 !} \frac{\partial^{6} V\left(S_{i}, t^{n}\right)}{\partial S^{6}}+\ldots .
\end{gathered}
$$

Substituting the above Taylor series expansions to the finite difference approximation in eqn (8) at a particular grid point $\left(S_{i}, t^{n}\right)$ shows that it is consistent to the original PDE [10][1] in eqn (7) through the consistency relation up to the terms $\delta t^{2}$ and $\delta S^{2}$

$$
\frac{\partial \tilde{V}(S, t)}{\partial t}-L_{B S} \tilde{V}=S_{t}
$$

where

$$
S_{t}=-\frac{\delta t}{2} L_{B S}\left(L_{B S} \tilde{V}\right)-\frac{\delta t^{2}}{6} L_{B S}\left(L_{B S}\left(L_{B S} \tilde{V}\right)\right)-\delta S^{2}\left(\frac{1}{3 !} r S \frac{\partial^{3} \tilde{V}}{\partial S^{3}}+\frac{1}{4 !} \sigma^{2} S^{2} \frac{\partial^{4} \tilde{V}}{\partial S^{4}}\right) .
$$

The solution $\tilde{V}$ of eqn (12) is in essence the exact solution of the finite difference approximation in eqn (8). As $\delta t \rightarrow 0$ and $\delta S \rightarrow 0$ eqn (12) reduces to the original BS PDE in eqn (7), and in this situation $\tilde{V} \rightarrow V$. As $\delta t$ exhibits a finite size which resembles a discrete trading time step, $S_{t}$ consists of correction terms of $\delta t$ and $\delta t^{2}$ of a recursive BS operator $L_{B S}$ acting on $\tilde{V}$ and higher order spatial derivatives of $\tilde{V}$ to correct the pricing function obtained by means of the BS PDE in eqn (7). 
Define $L=\sigma^{2} S^{2} \frac{\partial}{\partial S}+r$. Using the original BS PDE it is possible to derive the following four relations.

$$
\begin{gathered}
\frac{\partial}{\partial t}\left(\frac{\partial V}{\partial S}\right)-L_{B S}\left(\frac{\partial V}{\partial S}\right)-L\left(\frac{\partial V}{\partial S}\right)=0 \\
\frac{\partial}{\partial t}\left(\frac{\partial^{2} V}{\partial S^{2}}\right)-L_{B S}\left(\frac{\partial^{2} V}{\partial S^{2}}\right)-L\left(\frac{\partial^{2} V}{\partial S^{2}}\right)-L\left(\frac{\partial V}{\partial S}\right)=0 \\
\frac{\partial}{\partial t}\left(\frac{\partial^{3} V}{\partial S^{3}}\right)-L_{B S}\left(\frac{\partial^{3} V}{\partial S^{3}}\right)- \\
L\left(\frac{\partial^{3} V}{\partial S^{3}}\right)-L\left(\frac{\partial^{2} V}{\partial S^{2}}\right)-L\left(\frac{\partial V}{\partial S}\right)=0 \\
\frac{\partial}{\partial t}\left(\frac{\partial^{4} V}{\partial S^{4}}\right)-L_{B S}\left(\frac{\partial^{4} V}{\partial S^{4}}\right)- \\
L\left(\frac{\partial^{4} V}{\partial S^{4}}\right)-L\left(\frac{\partial^{3} V}{\partial S^{3}}\right)-L\left(\frac{\partial^{2} V}{\partial S^{2}}\right)-L\left(\frac{\partial V}{\partial S}\right)=0
\end{gathered}
$$

Note that solving eqns (15) and (16) provides knowledge of higher derivatives of $\tilde{V}$ which may be used in eqn (12).

\section{Discrete Trading and its Effect}

Let $\delta t$ be the discrete time step between two successive trading times. It is assumed that the discrete assest price changes according to the discrete backward difference equation

$$
\delta S=S_{i+1}-S_{i}=a \delta t+b \delta t^{1 / 2} \epsilon_{i+1}, i=0,1, \ldots
$$

where $\epsilon_{i}$ represents an independent Gaussian distributed variable. Here $a=\mu S$ and $b=\sigma S$. This equation is in essence the Euler discretisation of the continuous SDE defined in eqn (1). The incremental change of the option value when $\delta t$ is a finite value can be exspanded using Taylor series expansion as

$$
\begin{gathered}
\delta V=\frac{\partial V}{\partial t} \delta t+\frac{\partial V}{\partial S} \delta S+ \\
\frac{1}{2} \frac{\partial^{2} V}{\partial S^{2}} \delta S^{2}+\frac{1}{2}\left(\frac{\partial^{2} V}{\partial t^{2}} \delta t^{2}+2 \frac{\partial^{2} V}{\partial S \partial t} \delta S \delta t\right)+ \\
\frac{1}{3 !} \frac{\partial^{3} V}{\partial S^{3}} \delta S^{3}+\frac{1}{3 !}\left(\frac{\partial^{3} V}{\partial t^{3}} \delta t^{3}+3 \frac{\partial^{3} V}{\partial S^{3} \partial t} \delta S^{2} \delta t+3 \frac{\partial^{3} V}{\partial S \partial t^{2}} \delta S \delta t^{2}\right)+
\end{gathered}
$$




$$
\frac{1}{4 !} \frac{\partial^{4} V}{\partial S^{4}} \delta S^{4}+\frac{1}{4 !}\left(\frac{\partial^{4} V}{\partial t^{4}} \delta t^{4}\right) .
$$

Using eqn (17) to calculate $\delta S^{2}, \delta S^{3}$ and $\delta S^{4}$, substituting the results into eqn (18) and keeping terms upto $\delta t^{2}$ leads to

$$
\delta V=A \delta t^{1 / 2}+B \delta t+C \delta t^{3 / 2}+D \delta t^{2}
$$

where $A=b \epsilon \frac{\partial V}{\partial S}, B=\frac{\partial V}{\partial t}+\frac{1}{2} b^{2} \epsilon^{2} \frac{\partial^{2} V}{\partial S^{2}}+a \frac{\partial V}{\partial S}, C=a b \epsilon \frac{\partial^{2} V}{\partial S^{2}}+\frac{1}{6} b^{3} \epsilon^{3} \frac{\partial^{3} V}{\partial S^{3}}+b \epsilon \frac{\partial^{2} V}{\partial S \partial t}$. and $D=\frac{1}{2} \frac{\partial^{2} V}{\partial t^{2}}+\frac{1}{24} b^{4} \epsilon^{4} \frac{\partial^{4} V}{\partial S^{4}}+\frac{1}{2} a b^{2} \epsilon^{2} \frac{\partial^{3} V}{\partial S^{3}}+\frac{1}{2} a^{2} \frac{\partial^{2} V}{\partial S^{2}}+a \frac{\partial^{2} V}{\partial S \partial t}+\frac{1}{2} b^{2} \epsilon^{2} \frac{\partial^{3} V}{\partial S^{2} \partial t}$.

Use the same portfolio as defined in eqn (4), the incremental change of the portfolio can be written as

$$
\delta \Pi=\delta V-\Delta \delta S,
$$

and to offset the risk and eliminate the uncertainty in the option prices, one needs to compute the expected value of $\delta \Pi$ instead of using eqn(5). Hence one needs to use

$$
E[\delta \Pi] \equiv E[\delta V]-\Delta E[\delta S]=r(V-\Delta S) \delta t
$$

A suitable choice ol $\Delta$ in this case is needed. This could be achieved by minimising the variance of the portfolio. From eqn (20) one can obtain the variance of $\delta \Pi$ as

$$
\operatorname{var}[\delta \Pi]=\operatorname{var}[\delta V]+\Delta^{2} \operatorname{var}[\delta S]-2 \Delta \operatorname{cov}[\delta V, \delta S]
$$

It can be easily shown that the minimum variance of the portfolio occurs when $\Delta_{o p t}=\frac{\operatorname{cov}[\delta V, \delta S]}{\operatorname{var}[\delta S]}$. Let $\rho$ be the correlation coefficient between $\delta V$ and $\delta S$. Therefore $\Delta_{\text {opt }}$ can be written as

$$
\Delta_{o p t}=\frac{\rho \sqrt{\operatorname{var}[\delta V] \operatorname{var}[\delta S]}}{\operatorname{var}[\delta S]}
$$

Substituting the above optimal value to eqn (21) leads to

$$
\operatorname{var}[\delta \Pi]=\operatorname{var}[\delta V]\left(1-\rho^{2}\right)
$$

Substituting the above optimal value to eqn (20) leads to

$$
E[\delta V]-\rho \sqrt{\frac{\operatorname{var}[\delta V]}{\operatorname{var}[\delta S]}} E[\delta S]=r\left(V-\rho \sqrt{\frac{\operatorname{var}[\delta V]}{\operatorname{var}[\delta S]}} S\right) \delta t,
$$

which can be re-arranged to give the relation

$$
\frac{E[\delta V]-r V \delta t}{\sqrt{\operatorname{var}[\delta V]}}=\rho \frac{E[\delta S]-r S \delta t}{\sqrt{\operatorname{var}[\delta S]}} .
$$


In the case of discrete trading which means $\rho \neq 1$, the relation $E[\delta V]=r V \delta t$ must hold. This is used in the following analysis.

Note that in the present study $E[\epsilon]=0, E\left[\epsilon^{2}\right]=1, E\left[\epsilon^{4}\right]=3 ! !=3$ and the variance of the discrete random numbers is 1 . Using these results one obtains $E[A]=$ $0, E[C]=0$, and

$$
\begin{gathered}
E[B]=\frac{\partial V}{\partial t}+\frac{1}{2} b^{2} \frac{\partial^{2} V}{\partial S^{2}}+a \frac{\partial V}{\partial S} \\
E[D]=\frac{1}{2} \frac{\partial^{2} V}{\partial t^{2}}+\frac{3}{24} b^{4} \frac{\partial^{4} V}{\partial S^{4}}+\frac{1}{2} a b^{2} \frac{\partial^{3} V}{\partial S^{3}}+\frac{1}{2} a^{2} \frac{\partial^{2} V}{\partial S^{2}}+a \frac{\partial^{2} V}{\partial S \partial t} .
\end{gathered}
$$

Combining the results of the expected values and the relation $E[\delta V]=r V \delta t$ and keeping terms upto $\delta t^{2}$ leads to

$$
\begin{gathered}
\frac{\partial V}{\partial t}+\frac{1}{2} b^{2} \frac{\partial^{2} V}{\partial S^{2}}+a \frac{\partial V}{\partial S}-r V \\
+\delta t\left(\frac{1}{2} \frac{\partial^{2} V}{\partial t^{2}}+\frac{3}{24} \frac{\partial^{4} V}{\partial S^{4}}+\frac{1}{2} a b^{2} \frac{\partial^{3} V}{\partial S^{3}}+\frac{1}{2} a^{2} \frac{\partial^{2} V}{\partial S^{2}}+a \frac{\partial^{2} V}{\partial S \partial t}+\frac{1}{2} b^{2} \frac{\partial^{3} V}{\partial S^{2} \partial t}\right)=0 .
\end{gathered}
$$

Differentiating the above PDE one can obtain $\frac{\partial^{2} V}{\partial t^{2}}, \frac{\partial^{2} V}{\partial S \partial t}$, and $\frac{\partial^{3} V}{\partial S^{2} \partial t}$ which are then substituted back to the PDE to give

$$
\frac{\partial V}{\partial t}-L_{B S} V=\Gamma_{D}
$$

where

$$
\begin{aligned}
& \Gamma_{D}=\delta t\left\{\frac{1}{2} \sigma^{4} S^{3} \frac{\partial^{3} V}{\partial S^{3}}+\left(\frac{1}{2} \sigma^{2} S^{2} \mu-\frac{1}{2} \sigma \mu S+\frac{1}{4} \sigma^{2} S^{2} r\right) \frac{\partial^{2} V}{\partial S^{2}}\right. \\
& \left.+\left(\frac{1}{2} \mu^{2} S+\frac{1}{2} \mu S r\right) \frac{\partial V}{\partial S}+\frac{1}{2} r L_{B S} V\right\}=0 .
\end{aligned}
$$

This is the source term introduced to the pricing PDE when discrete trading takes place. Note that $\Gamma_{D}$ becomes larger as $\delta t$ increases.

\section{Conclusion}

The importance of discrete trading strategy is discussed. Two aspects of discrete trading are discussed, one due to the finite difference discretisation of the BlackScholes PDE and the other due to the discrete analog of the SDE governing the dynamics of the asset. In both cases modified source terms are derived leading to modified Black-Scholes models. As $\delta t$ becomes larger the modified source terms become significant. The modified source terms tend to zero as $\delta t$ tends to zero which 
reduces to the pricing obtained by continuous trading.

\section{REFERENCES}

1. U.M. Ascher (2008). Numerical Methods for Evolutionary Differential Equations. SIAM, ISBN 978-0-898716-52-8.

2. F. Black, M. Scholes (1973). The pricing of options and corporate liabilities. Journal of Political Economy, 81, 637-654.

3. D. Crann, A. J. Davies, C.-H. Lai, and S. W. Leong (1998). Time domain decomposition for European options in financial modelling. Editors: Mandel et al, Domain Decomposition Methods 10, American Mathematical Society.

4. E. Derman (1999). When you cannot hedge continuously, the corrections of BlackScholes. Risk, 1, 82-85.

5. B. Dupire (1994). Pricing with a smile. Risk, 7, 18-20.

6. C.-H. Lai, A. K. Parrott, S. Rout (2005). A distributed algorithm for European options with nonlinear volatility. Computers and Mathematics with Applications, 49, 885-894.

7. C.-H. Lai, A.M.S. Ribeiro, N. Kokulan (2017). Alternative parallel strategies for linear and nonlinear PDEs in option pricing. In STRIKE - Novel Methods in Computational Finance, eds. Ehrhardt, Günther, ter Maten Springer, 433-441.

8. S. Mayhew (1995). Implied volatility. Financial Analysts Journal, 51 (4), 8-20.

9. B. Øksendal (2000). Stochastic Differential Equations: An Introduction with Applications, 5th edition. Springer, ISBN 3-540-63720-6.

10. G.D. Smith (1985). Numerical Solution of Partial Differential Equations: Finite Difference Methods, 3rd Edition. Oxford University Press, ISBN 0198596502.

11. S.-H. Tan (2017). Towards efficient nonlinear option pricing. PhD Thesis, University of Greenwich.

12. S.-H. Tan, C.-H. Lai (2017). Newton based solvers for nonlinear PDEs in finance. In STRIKE - Novel Methods in Computational Finance, eds. Ehrhardt, Günther, ter Maten Springer, 229-242. 
13. O. Vasicek (1977). An equilibrium characterisation of the term structure. Journal of Financial Economics, 5, 177-188.

14. P. Wilmott (1994). Discrete charms. Risk Magazine, 7 (3), 48-51.

15. P. Wilmott, S. Howison, J. Dewynne (1995). The Mathematics of Financial Derivatives. Press Syndicate, ISBN 0-521-49789-2. 